OPEN ACCESS

Edited by:

Chris Duvall,

University of New Mexico,

United States

Reviewed by:

Pablo Torres-Lima,

Metropolitan Autonomous

University, Mexico

Michael Hardman,

University of Salford, United Kingdom

*Correspondence:

Bianca Cavicch bianca.cavicchi@nibio.no

Specialty section:

This article was submitted to

Urban Agriculture,

a section of the journal

Frontiers in Sustainable Food Systems

Received: 27 April 2021

Accepted: 04 October 2021

Published: 03 November 2021

Citation:

Cavicchi B and Hegnes AW (2021)

Scoping Out Elements of Sociocultural

Adaptation in European Urban

Agriculture

Front. Sustain. Food Syst. 5:701160.

doi: 10.3389/fsufs. 2021.701160

\section{Scoping Out Elements of Sociocultural Adaptation in European Urban Agriculture}

\author{
Bianca Cavicchi* and Atle Wehn Hegnes \\ Division of Food Production and Society, Norwegian Institute of Bioeconomy Research, Ås, Norway
}

This paper explores and sheds light on the elements, complexity, and dynamics of sociocultural adaptation to innovation and climate change in European Urban Agriculture. We use a scoping-exploratory review to search and unveil elements of sociocultural adaptation (SCA) in the existing literature on European urban agriculture. We categorize these elements into three main categories. This categorization can inform and be further explored, operationalized, and developed in new case-study-based research and serve as a starting point to better understand social adaptation to innovation and climate change in urban contexts, and beyond. Key results draw attention to (a) socio-technical and socio-ecological innovations as critical to sociocultural adaptation to innovation and climate change (b) some elements of SCA identified through the scoping review seem more central than others for the adaption process (c) we are left with the question of whether we need to bridge social science with biology sciences, such as human behavioral biology and neurobiology to find the answer to deeper questions about SCA.

\footnotetext{
Keywords: European urban agriculture, sociocultural adaptation, scoping review, sustainable food systems, Farm-to-Fork strategy
}

\section{INTRODUCTION}

The European Green Deal and the Farm-to-Fork strategy are current and crucial descriptions and plans for future, inclusive and systemic ways of organizing and structuring sustainable food production (European Commission, 2020). As part of the same sustainable evolution, European cities are increasingly becoming loci for socio-technical experimentations, including technologies, novel modes of organization, food systems, networking, and collaboration to help societies adapt and become more resilient to climate change. Cities are, however, also known to have a history of distancing natural ecosystems and biodiversity problems (Cicekli and Barlas, 2014; Classens, 2015; Durrant et al., 2018; Giacchè and Porto, 2018; Boossabong, 2019; Hennchen and Pregernig, 2020; Schoen et al., 2020) and the current renaissance of the phenomenon of Urban Agriculture (UA) twists the cities historical reputation and shows how to re-kindle nature, society and technologies within sustainable urban food systems (Thornbush, 2015; Sanyé-Mengual et al., 2016; Berthet and Hickey, 2018; Giacchè and Porto, 2018). A few Horizon Europe projects recently demonstrate this 
new interest for cities as core contributors to sustainability and resilience, e.g., SiEUGreen ${ }^{1}$, Efua ${ }^{2}$, Urban Allotment Gardens ${ }^{3}$, and fusilli-project. ${ }^{4}$

The pathway toward urban sustainable food systems will require changes and adjustments in interlinked social, technological, and biological domains (Zimmerer et al., 2021). Understanding this complex of sociocultural adaptation (SCA) is already a repeated and general theme in the literature on UA and socio-ecological resilience (Zimmerer et al., 2021). Two recent literature review studies, namely Topal et al. (2021) and Gulyas and Edmondson (2021), may suit as examples of this "new" avenue of UA research. Whereas, the first contribution represents an example of a review mapping socio-psychological perspectives on understandings and behavior of urban sustainability, the second contribution systematically reviews the literature on UA in the global north and its impact on city resilience through UA. Additionally, both studies develop conceptual models based on their reviews. Topal, Hunt and Rogers conclude that their framework, consisting of three layers of clustered components, is needed for an integrative approach to urban sustainability understanding and behavior. The components are developed and presented in a model (p. 26), consisting of (1) internal socio-psychological determinants, (2) personality traits, and (3) influencing external factors such as social, cultural, economic, and institutional factors. In the second review, Gulyas and Edmondson find that UA's success in increasing city resilience is determined by five factors developed and illustrated as a model (p. 12). Common for both studies is the identification of relevant literature and their conceptual models that contribute to the theoretical-methodological toolbox to understand different aspects of UA.

Whereas, knowledge on socio-psychological and institutional components for sustainable UA in Europe has been covered in the reviews mentioned above and elsewhere, few have identified and systematized the complexity of sociocultural adaptation in European UA. There is a variety of definitions of social adaptation and sociocultural change, building on the legacy of forerunners within this field (Bristol, 1915; Ogburn, 1922; Sorokin, 2006). However, as we shortly will return to, we initially defined "sociocultural adaptation in UA" as changes or adjustments in critical dimensions, such as culture, practices, norms, perceptions and frames (Woolston, 1917; Cote and Nightingale, 2012). Against this background, and based on a scoping review, we explored some of the elements, complexity, and dynamics of SCAs in the European Urban Agriculture. Our results shed light on the blind spot of SCAs in the research on UA and contribute to filling the conceptual-methodological toolbox to understand the nuances and complexity of this emerging phenomenon.

In the remainder of the paper, we proceed as follows: first, we describe the methodology and the process we followed in conducting the literature review and identifying and defining

\footnotetext{
${ }^{1}$ https://www.sieugreen.eu/

${ }^{2}$ https://efua.eu/

${ }^{3}$ https://www.urbanallotments.eu/

${ }^{4}$ https://fusilli-project.eu/
}

the themes of sociocultural adaptation. Second, we describe, analyze, and summarize the results from the review and the identified literature categories and their related set of identified elements of sociocultural adaptations. Third and finally, we discuss the results and conclude with some methodologicaltheoretical implications.

\section{METHODS}

Scoping reviews are ideal for mapping out a body of literature on a given topic (Munn et al., 2018), such as SCA in European UA. This relatively new tool, for which there is not a universal methodological approach yet, are intended to help examine emerging and heterogenous evidence when it is still unclear what other, more specific questions can be posed and valuably addressed by a more detailed systematic review (Pham et al., 2014).

In our scoping review, we built on the six-stages iterative process proposed by Arksey and O’Malley (2007), Pham et al. (2014) and adapted it to the needs of the study. The review was conducted to scan the vast body of existing literature on European UA and explore what is known about SCA. The six stages included:(1) identifying the research question, (2) identifying relevant studies, (3) study selection, (4) charting the data, (5) collating, summarizing and reporting the results, and (6) an optional consultation exercise. We complemented this with an abductive approach for a continuous dialogue between the researcher, theories and concepts, and empirical data (i.e., articles in this case), and therefore the adaptation of the search as research results came through (Alvesson and Sköldberg, 2009) (Figure 1).

At the start of the research process, we developed a protocol for the scoping review as a guideline for steps to undertake. However, as we adopted an abductive approach, the protocol was revised along with the findings and needs that emerged during the scoping review process. Figure 1 below shows the stages of the scoping review process. We also included an additional step that followed the first round of peer-review (Table 1).

\section{Research Questions}

The scoping review was initially based on a two-fold research question: (i) What is known about SCA in the European research literature on Urban Agriculture? (ii) How can the formalization of this knowledge contribute to understanding SCA in food system sustainability transitions? We defined the research question to allow for the breadth of the review coverage, although aware that we would not cover all contexts and cases in Europe. Therefore, these research questions aimed to provide enough literature to unveil elements that could contribute to understanding the complexity and dynamics of sociocultural adaptation in UA.

\section{Data Sources-Relevant Studies}

As already mentioned, we started the review by defining "sociocultural adaptation in UA" as changes or adjustments in critical dimensions, such as culture, practices, norms, perceptions and frames (Woolston, 1917; Cote and Nightingale, 2012). 


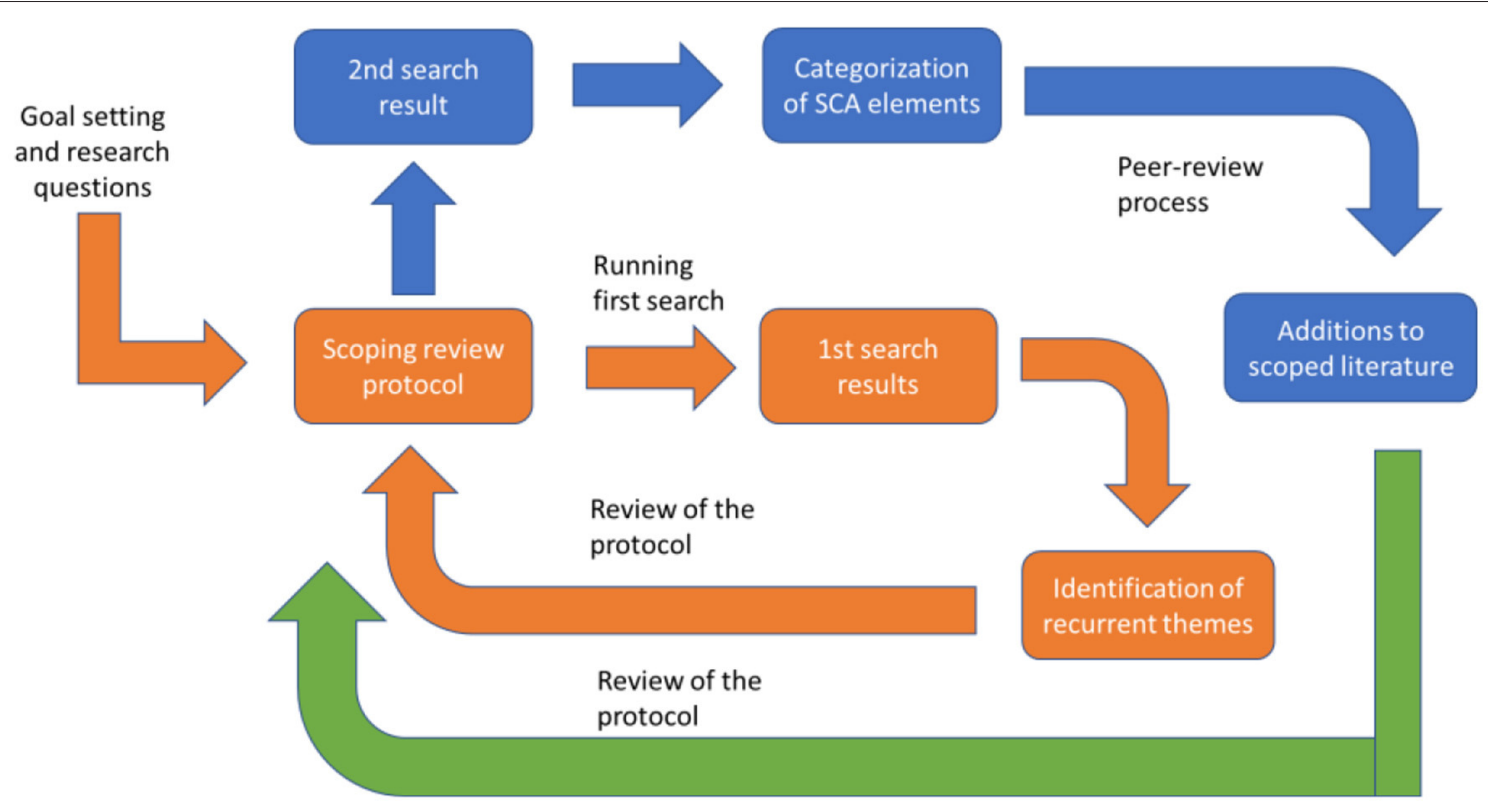

FIGURE 1 | Scoping review process.

TABLE 1 | Scoping review protocol.

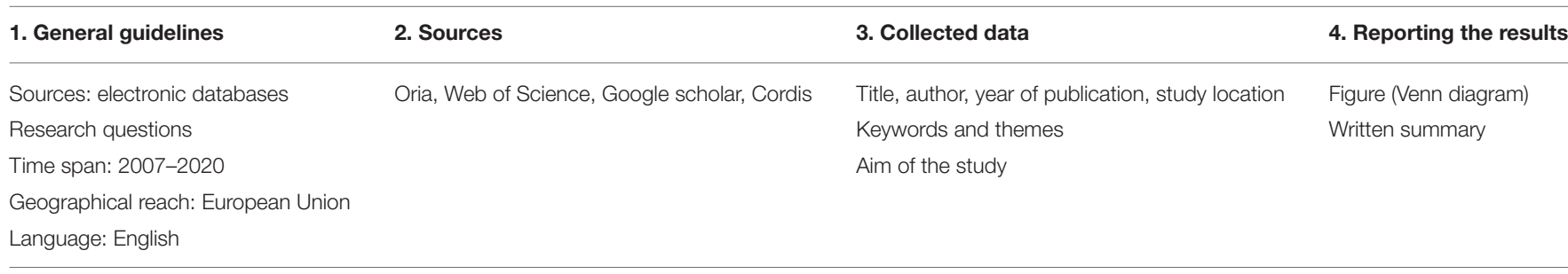

Further, we defined the spatial and temporal boundaries of our search. Finally, we searched the research concerning UA in Europe from 2007 to 2020. The rationale behind this choice is rooted in European policy developments. Increased interest in urban agriculture became relevant in the European debate approximately in the early 2010s (Curry et al., 2014; McEldowney, 2017).

Further, we started the review process using the University of Oslo's server-Oria, ${ }^{5}$ and searched for all types of publications, from peer-reviewed to unpublished work (i.e., gray literature). The search query included terms considered by the authors to describe best the aim of the scoping review and the definition of sociocultural adaption in UA. Therefore, we started with a combination of the following keywords:

- UA

- UA, culture, technology,

- UA, Sustainability Transitions (ST)

- UA, ST, technology,

${ }^{5}$ https://bibsys-almaprimo.hosted.exlibrisgroup.com/primo-explore/search?vid= UIO\&lang=en_US
- UA, culture,

- UA, adaptation

- UA, adaptation, culture,

- UA, culture, practice,

- UA, technology, adaptation,

- UA, ecological transition

- UA, transition, technology, practices.

We made the a priori decision to screen the first 30 items for each search (as sorted by relevant by Oria), excluding all the items that did not focus on the European context. We believed that more items would divert the focus from the key aspects of UA that we wanted to explore. We ran 13 first searches to give scope and breadth to the review and understand the key trends in UA literature from SCA's perspective. We also decided to search in google scholars manually.

At first glance, most of the UA studies in the different combinations of searches were carried out in the global south (e.g., Asia-Vietnam, India, China, Japan-Africa, US, Australia, and Canada). Comparatively, few studies were done in Europe on SCA to new urban agriculture technologies, considering aspects such as institutions, sustainability 
TABLE 2 | Inclusion and exclusion criteria ${ }^{\mathrm{a}}$.

\begin{tabular}{|c|c|}
\hline Inclusion & Exclusion \\
\hline $\begin{array}{l}\text { Peer-reviewed articles published in } \\
\text { English on European case studies }\end{array}$ & $\begin{array}{l}\text { Peer-reviewed articles not published } \\
\text { in English and dealing with cases } \\
\text { outside Europe }\end{array}$ \\
\hline Year 2007-2020 & Any study before 2007 \\
\hline Studies dealing with UA. & Studies not dealing with UA. \\
\hline
\end{tabular}

${ }^{a}$ The table with inclusion and exclusion criteria: year, geographical location, studies not linking to urban agriculture, studies dealing with climate adaptation rather than adaptation to innovation (i.e., technology).

transitions and technology, sociocultural conditions, and mental frames.

\section{The First Selection of Studies}

After reading these first selected studies, we recognized that those not set in the EU dealt with climate change adaptation rather than focusing on UA specifically or addressed urban transitions and sustainability issues, not from a UA perspective (Table 2).

We then went back to our research question and goal to decide on the next steps, extrapolate the recurrent themes, and match them with our goal and research question. Based on the analysis of the text of the papers and the word searches that we had run, we saw that there were recurrent concepts in the paper, such as community actions, technologies, urban infrastructure design. Therefore, we categorized the recurrent themes that emerged during the first search as:

1. Studies focusing on social adaptations community actions, policies, and social resilience

2. Studies focusing on social adaptations on socio-technical transitions and urban innovations

3. Studies focusing on social adaptations in high-tech and lowtech adoption

4. Studies focusing on social adaptations in urban infrastructure design

5. Studies focusing on social adaptations in urban ecosystem services

At this point, we decided to run a second round of searches. Covering several disciplines and regions was important in our scoping review. Therefore, we decided to use Web of Science. Web of Science is a cross-disciplinary, international article search engine that gives access to multiple databases, thus providing comprehensive citation data for a range of different academic disciplines. We searched in which other studies the selected articles under each category were quoted. We believed that such a narrowed search would yield more selected and relevant searches to meet the research questions and goal of the scoping review.

\section{The Second Selection of Studies and Data Charting}

This second search supported the validation and refinement of the previously identified recurrent themes in the literature. Notably, we decided to cluster them into three categories, i.e., (1) studies focusing on social adaptations in community actions, policies, and social resilience; (2) studies focusing on social adaptations in socio-technical transitions and urban innovation; (3) studies focusing on social adaptations in high-tech and lowtech UA. We combined literature on community actions, policies, and social resilience into one category because UA has three strongly interlinked dimensions. Local initiatives and linked upscaling processes influence and are influenced by policies and cannot be separated if one wants to understand SCAs. Within each category, we then identified some critical SCA elements. We inferred these elements based on evidence gathered through the literature review.

\section{Additions Post First Peer-Review}

We added some new and essential studies based on the reviewers' suggestions during the first round of review. We also scanned European funded projects about Urban Agriculture and Urban Gardens in the "Cordis database." We selected the most relevant of them based on the paper's primary focus, i.e., sociocultural adaptations in UA, thus excluding projects dealing with natural science aspects. Our search yielded four projects already mentioned in the introduction: SiEUGreen ${ }^{6}$, Efua $^{7}$, Urban Allotment Gardens ${ }^{8}$, and fusilli-project ${ }^{9}$. From the projects' website, we scanned the published literature and other contents (not all projects have already available publications), and we added them to the three categories of elements of SCA.

\section{REPORTING THE RESULTS: ELEMENTS AND DYNAMICS OF SOCIOCULTURAL ADAPTATION-RESULTS AND ANALYSIS}

This section reports and summarizes the results under three literature themes of SCAs, consisting of different adaptation strategies and practices. The strategies and practices contribute to the analytical-conceptual toolbox for understanding the dynamics of sociocultural adaptations in European UA, further elaborated in summation sections and the concluding discussion.

The key results are shown in the Venn diagram below (Figure 2). The diagram shows that the different categories overlap, thus seemingly indicating that some of these elements are more central than others. However, as the study is explorative, the elements and dynamics need further research, notably through theoretical or case study research.

\section{Social Adaptations in Community Actions, Policies, and Social Resilience}

Most of the studies included in this category deal with community actions, policies, and social resilience (Barthel and Isendahl, 2013; Hearn et al., 2014; Taylor and Lovell, 2014; Camps-Calvet et al., 2015; Corcoran and Kettle, 2015; Mancebo, 2016; Partalidou and Anthopoulou, 2017; Russo et al., 2017; Hennchen and Pregernig, 2020; Schoen et al., 2020). Within the domain of urban agriculture, authors often emphasize

\footnotetext{
${ }^{6}$ https://www.sieugreen.eu/

${ }^{7}$ https://efua.eu/

${ }^{8}$ https://www.urbanallotments.eu/

${ }^{9}$ https://fusilli-project.eu/
} 


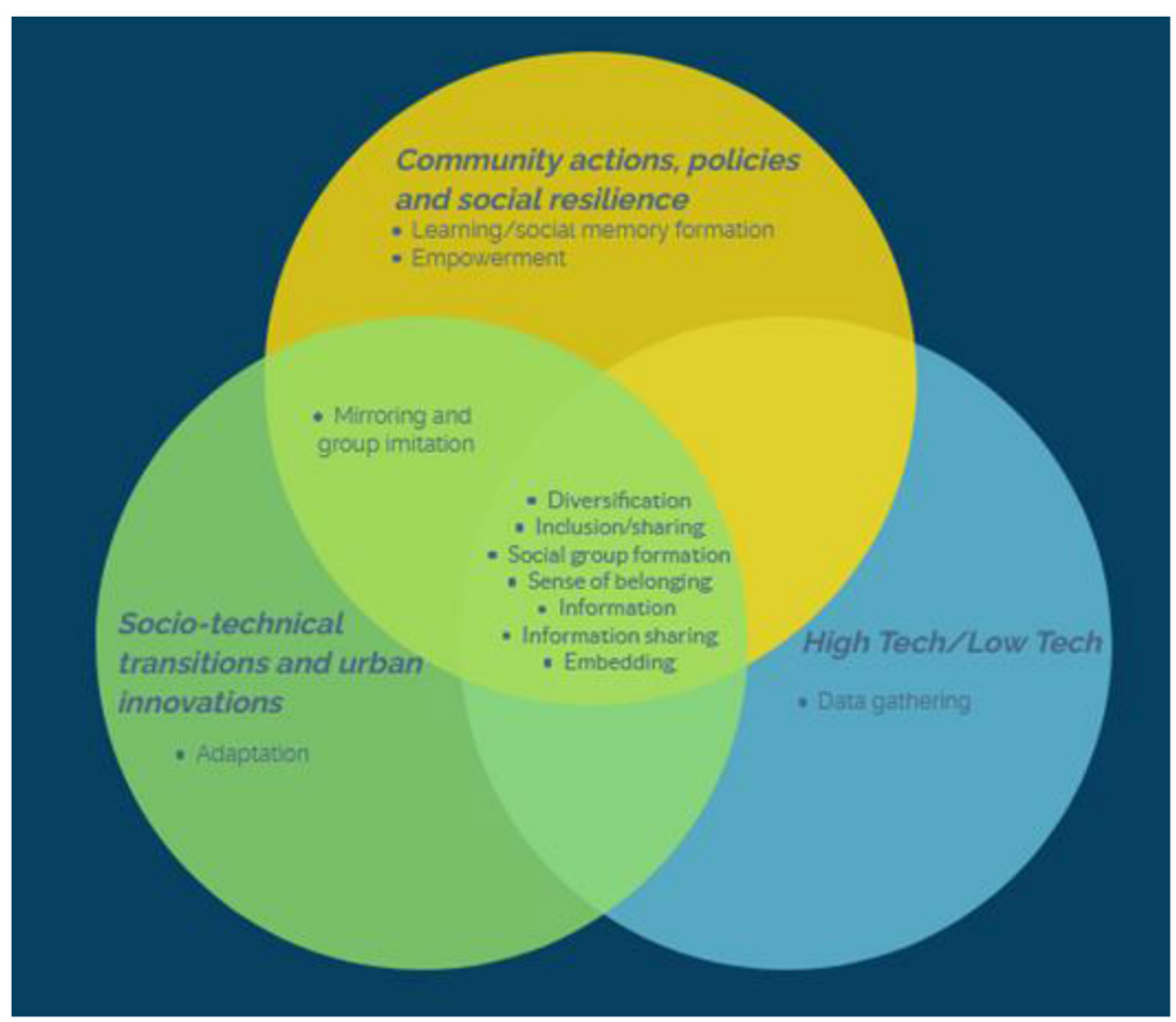

FIGURE 2 | Venn diagram sociocultural adaptation elements in literature themes on UA\&SC.

community actions or local initiatives to describe new ways of organizing food production, social engagement, resistance and contestation, social inclusion, managing public spaces and dealing with social and environmental justice. Especially interesting is the paper by Camps-Calvet et al. (2015). It describes urban gardening initiatives in 27 European cities where, particularly in Southern European countries, urban gardens and agriculture have become forms of resistance through collective actions that can boost social cohesion and social justice. The paper mentions a few consequential categories to build up resilience that can fall within those that we have listed in this category of social adaptations, i.e., learning from past crises, learning from various knowledge sources, nurturing cultural diversity, building knowledge into practices and habits, nurturing social cohesion and policy developments (Camps-Calvet et al., 2015). On the latter, we have found that policies have a crucial role in facilitating or hampering the blooming of these local actions.

Moreover, policies can also enable or hamper social resilience. Social resilience can here be defined as the capacity of communities to face challenges either by adapting or transforming their practices, institutions, norms and frames. Notably, social resilience is "the ability of groups or communities to cope with external stresses and disturbances as a result of social, political, and environmental change (Adger, 2000)" (Cote and Nightingale, 2012). In these studies, the focus is on the local, cityscale. However, many also relate to the national scale concerning the role of policies. Therefore, this is an example of cross-scales linkages that shape the SCA process.

Based on the studies included in this literature category, we inferred four elements of the SCA, i.e., learning, and social memory formation; diversification, inclusion and sharing; empowerment.

\section{Learning/Social Memory Formation}

Barthel and Isendahl (2013) draw on the social-ecological resilience approach, which identifies two key features to strengthen the resilience of social-ecological systems, i.e., diversity and learning/memory. The social-ecological resilience concept was introduced in forest ecology to signify the capacity of systems to absorb shocks and tackle stressors over time to bounce back or reorganize without losing the necessary functions. The diversity of species is crucial because they respond differently to shocks and stressors as memories of past events are stored differently in their genetic code, thus increasing the system's ability to endure disturbances. In social terms, learning, hence memory, is a pivotal element to ensure that communities can use past experiences to respond to new challenges. Barthel and Isendahl apply these ecological concepts to understand adaptation in urban contexts concerning food systems adaptation. Besides considering the diversity of food sources (e.g., urban agriculture) and other similar aspects, they also investigate social memory as a dimension of city resilience. They argue that social/collective memory carriers are practices, 
norms, and institutions, besides DNA and genetic code. "Here we choose the concept of social-ecological memory since it captures vital relations between humans and living ecosystems that affect the ability of people to respond to disturbance (Folke et al., 2003), defined as how knowledge, experience, and practice about how to manage a local ecosystem and its services are retained in a society and revived and transmitted through time (Barthel, 2010) (Barthel and Isendahl 2013: p. 226). The two authors stress some exciting and essential aspects concerning the primary goal of this study. Notably, SCA to changes in technologies, products and processes is affected. It will affect social memory in its various forms, the diverse types of urban agriculture and subsequent choices of high-tech and low-tech options, the spatial distribution of urban agriculture communities in a continuous learning feedback loop. In line with these arguments, Partalidou and Anthopoulou (2017) stress that some gardeners see urban gardens and agricultural practices as part of our collective and rural past that has been swept away by city life. Moreover, urban gardens provide an opportunity for people to learn new skills and acquire new knowledge. These dynamics linked to past and future ideals and ways of living indicate and support the assumption that urban agriculture can build new or renewed social memory.

\section{Diversification}

Other studies enrich the social-ecological narrative based on social memory and its carriers without using the same theoretical approach. For instance, authors such as Corcoran and Kettle (2015) draw on the idea that urban agriculture, in its various forms, creates a public space where sharing, inclusion and trust are strengthened within a context of equal social interaction. Often urban agriculture sites are open to everyone, democratic and with no "special" criteria for entrance selection. "Specifically, we argue that allotment sites produce an inclusive and socially cohesive notion of the public. This is possible because of the creation of a shared politics of place-a commitment to cultivation that is premised on individual labor carried out in a common cause, mutually agreed tacit rules of engagement and tolerance of diversity." Urban agriculture thus becomes a "sharing" and "inclusive" space where learning is enabled through trust and equality of conditions (Corcoran and Kettle, 2015: p. 1218). Thus, new collective knowledge, norms, practices, habits and rituals are created and habitualized within the urban agriculture community (situated, local features, and practices). Camps-Calvet et al. (2015) emphasized that in their European case studies, they found that urban gardens participants drew on diversified sources of knowledge, i.e., local and traditional ecological knowledge, but also experimental (e.g., laboratory of agricultural experimentation) (p. 427). Based on Barthel and Isendahl, we could hypnotize that this habitualization of new practices, norms, rituals, and habits may reinforce the social memory of the group and the city, thus reinforcing its ability to adapt to innovations.

\section{Inclusion/Sharing}

The study of Celata and Coletti (2018) contributes to the understanding of social resilience in UA by providing insights on community gardens as a form of self-regulation, community building and social inclusion, environmental justice, and social and political engagement (situated, local practices). "[...] the relationship between policymaking and community gardening is inherently problematic. The voluntarism, self-organization, variety and multidimensionality of community gardening is in fact hardly compatible with the regulatory anxiety, isomorphic pressures and sectorialism that most policy schemes inevitably imply." (p. 17). The authors stress that the members of community gardens often seek some form of political recognition and/or institutional support. Policymakers respond either by wanting to over-regulate the conditions that allow free community gardens, thus somehow restricting the types of possible ways of organizing community garden initiatives, or by directly promoting and diffusing the experience. The political responses to community garden initiatives will, on the one hand, impact the diversity dimension of resilience, either by restricting or increasing the number of different community gardens forms. On the other hand, these political responses will also shape the form and content of social memory and how social memory will be passed from a generation to another. Alongside, the study of Hennchen and Pregernig (2020) (but also Schoen et al., 2020), which suggests that local political and policy actions concerning urban food production can simultaneously foster (i) policy integration; (ii) more democratic processes as per including the local co-creators into decision-making processes and influence agenda-setting; provide the necessary infrastructures (e.g., local plots for gardening, kitchens and other things); (iii) broadening the participants' portfolio to others than the politicized or already aware and include people with the different sociocultural background. Camps-Calvet et al. go in a similar direction and "[...] suggest that urban gardens enhance mutual support structures" (p. 428).

Schwab et al. (2018) stress that Urban Agriculture has generally been framed within "models in circulation" of policy frames and policy designs. Notably, frames of UA for subsistence and contestation of current socio-economic arrangements and reclaim of the right to urban land. These policy frames result from the power relations and local cultural and other contextual conditions (e.g., environment, climate, economy, habits, norms, values etc.) that determine which ones will become dominant in the policymaking. This is again reflecting the spatial dimension of social adaption in UA.

One of these consequences is a reduced diversification of UA initiatives regarding goals, structures, political agendas, environmental justice, etc. Therefore, power issues and cultural conditions are two key factors that influence social resilience (in the dimensions of diversification and social memory).

\section{Empowerment}

From a different perspective, as Hearn et al. (2014) point out, social media can play a crucial role in building the group identity, thus strengthening the sense of belonging, equality and sharing. Hearn et al. argue that agriculture is inherently social, but without forgetting the ecological dimension (on this, they are in line with Barthel and Isendahl, 2013). Thus, social factors have a central role in boosting community building and social connectivity in 
developing sustainable and adaptive urban agriculture systems. "Within this context, information and communication technologies are innovations that can boost greater community engagement but inhibit change" (p. 203). ICTs foster connection between people, often in real-time, thus yielding a tremendous potential impact on consumption and production practices, preferences, habits and decisions [33 p. 24]. Communication technologies operate through social networks. Social networks facilitate and accelerate information transfer going beyond institutional aspects, thus using more horizontal channels. Communication technologies and social networks show the porosity and flexibility of spatiality and temporality, i.e., these technologies can boost the span of new social practices adoption over long distances and across scales. "Biggs et al. (2010) suggest further that ICTs have a central role in the move from a dangerous overdependence on centralized models of food, energy, water and transport systems to a more 'distributive' model of critical infrastructure provision: adaptive, localized, open and network based." [25 p. 2030]. Distributive systems are expected to be more resilient to change and ensure more economical, social, and ecological sustainability.

\section{First Summation}

First, we see how these four elements are interrelated and interdependent in a spatiotemporal perspective. We assume that one dynamic without the other could not lead to social resilience. Second, the diversification element is left unexplored in the reviewed papers. Based on what we read, we believe that diversification includes diverse actors and stakeholders in urban agriculture initiatives and actions that can influence policymaking. By diversifying actors, it will be possible to mitigate the effects of path dependency that social memory formation might generate and ensure that new norms, practices, rituals, narratives, and frames are the most inclusive.

Additionally, a diversified social group linked to urban agriculture would increase the extensiveness of social empowerment. Fourth, these four dynamics are particularly crucial in urban agriculture. As noted by the authors, UA often brings together people with different social, economic, cultural, and cognitive backgrounds, thus holding the potential to create new social rituals, norms, habits, and values. Thus, these dynamics are interrelated in that if UA initiatives are not inclusive, there will not be enough diversification of ideas, frames, tools, and strategies to cope with current challenges and the adoption of new technologies. Empowerment will also be impaired if there is no diversification of people in the UA social network (Figure 3).

\section{Social Adaptations in Socio-Technical Transitions and Urban Innovation}

Most of the studies we have classified within this category explain how local initiatives or niche level innovations (or grassroots innovations) can lead the transition to a more sustainable and greener urban society (Corcoran and Kettle, 2015; Cvejić et al., 2015; Huston et al., 2015; Mancebo, 2016; Barnes et al., 2018; Berthet and Hickey, 2018; Durrant et al., 2018; Fastenrath and Braun, 2018; Gorissen et al., 2018; Oda et al., 2018; Jürkenbeck et al., 2019; Sanyé-Mengual et al., 2019).

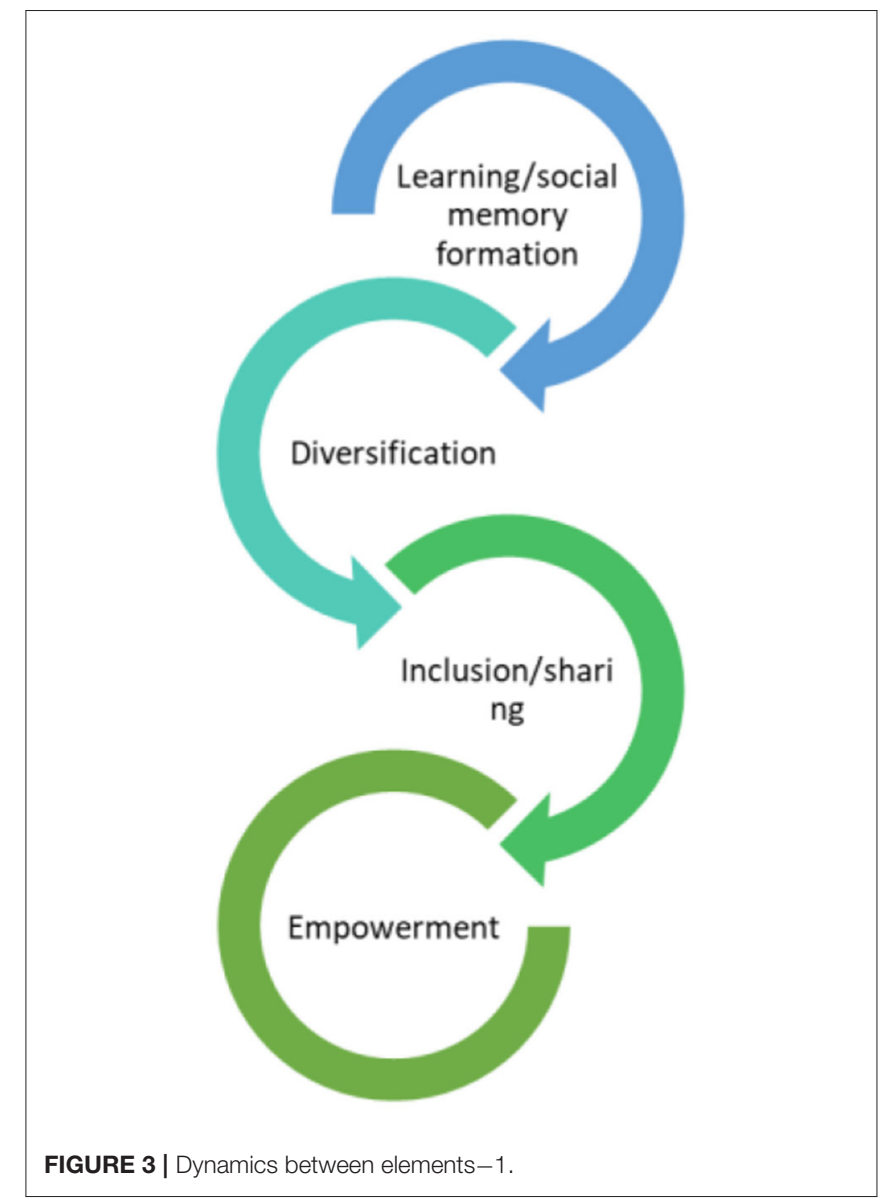

We have learned that building a collective "urban sustainability" narrative, which capitalizes on fundamental societal values, inclusion, and involvement of various actors is crucial for building synergies and networks of initiatives, diffuse and embed new practices, perceptions and routines into existing structures. We have also learned that sustainable innovation initiatives in cities often rely on resources from outside the urban context (e.g., energy, resources, water, food, sewage plants, and many commuters/urban workers). The urban metabolism is made of fluxes that involve outer areas, such as peri-urban, suburban, and rural areas that provide resources and services to the city. Thus, urban sustainability policies and initiatives should consider local ownership and these fluxes of people, resources and goods in the rural-urban continuum (Mancebo, 2016). The socio-technical transition perspective entirely relates to the spatiotemporal dimensions, as these scholars' reason for diffusion and upscaling of new practices, behaviors, norms, and technologies.

In the last decade, cities worldwide have been laboratories for various transition initiatives that include UA solutions (e.g., urban gardens, vertical agriculture, low-carbon energy solutions, and so on). "Sustainability initiatives often sprout from the local level, that is why they are often termed grassroots initiatives, and experiment with new governance arrangements, or technological, organizational or social innovation" (Gorissen et al. 2018: p. 
173). These initiatives are often the result of bottom-up and community actions (e.g., cities in transition or permaculture initiatives) or more structure and top-down actions such as policy and planning pilot programmes. They aim to transform lifestyles, practices, routines, and perceptions to respond to global sustainability challenges and effects on local sustainability. We list four SCA elements in this category, i.e., mirroring and group imitation; social group formation, sense of belonging, and adaptation.

\section{Mirroring and Group Imitation}

Most of the studies within this category focus on local actors and networks in the acceleration phase of the urban transitions. The acceleration phase occurs when grassroots (or niche level) innovations (thus sociocultural, economic, ecological, and institutional changes) start to be diffused, upscaled and embedded into the context they develop. In this phase, "replication" by other actors and "partnerships" (i.e., ability to build collaborations and exploit synergies between different actors' competencies and skills) are crucial for the urban transition initiative to exit the niche phase and become a diffused practice.

\section{Social Group Formation}

In this phase, the action potential of building dense and longlasting social networks that can facilitate knowledge acquisition and transfer, effective governance, inclusion and participation and resourcemobilization is essential to the acceleration and upscaling of local initiatives. Berthet and Hickey (2018) indicate that network managers should go beyond a pure focus on the experts' innovative solutions to include more and potentially conflicting and divergent ideas to give way to the best and most locally embedded solutions. This depends on the leadership and management of the network to ensure cooperation and smooth communication among diverse sets of actors.

\section{Sense of Belonging}

The capacity to replicate and establish partnerships also seem to differ based on the sector(s) involved in the transition process. Agriculture and food-related transitions seem to be more easily and quickly replicated within the geographical boundaries of the same city, compared to the energy and waste-related transition processes. This may be due to the infrastructural and value chains structure, which, in the former case, tend to stay local while, in the latter case, go beyond local boundaries. Partnering is fundamental for building trust within and between initiatives and upscale (i.e., involving new participants) the transition process. Partnering can also be instrumental for lobbying local governments in changing local institutional structures (e.g., regulatory schemes). Crucial to the diffusion of transition initiatives is using (or instrumentalize) available multi-level support schemes to boost local initiatives. It is also crucial to capitalize on the momentum and create societal narratives with emerging core values such as helping the vulnerable, boosting social inclusion and environmental justice, providing safe, sustainable, and organic food for all. The sense of belonging capitalizes on the last element of social network formation and the practices that allow replication and partnerships formation. These elements have a spatial and temporal dimension, i.e., they imply a local dimension that is upscaled and a time period within which these elements unfold.

\section{Adaptation}

Further, embedding the sociocultural and institutional changes of transition initiatives into new governance structures or by transforming old ones (e.g., new decision-making processes, new urban decision-making platforms, new forms of democracy, e.g., sociocracy) can increase the pace of change and diffusion of sustainable practices (Spatiotemporal dimension). However, there can be many obstacles to the fulfillment of the sustainability transition process, incl. corruption, political disagreement, lack of public trust, lack of funding, community polarization, displacement and resources gold rush and so on (patterns of behavior-practices). Huston et al., 2015 remind us that " $a$ more tempered approach, involving territorial foresight, debate, local engagement, institutional collaboration, project scrutiny, and smart finance." (p. 68). Mancebo et al. advocate for more focus on social innovation, inclusion, and participation to build an urban sustainable transition pathway.

\section{Second Summation}

In this category, we have learned that sustainability transitions are long-term processes within which many different elements operate to accelerate or slow down the societal transformation across scales (spatiotemporal dimensions). The authors in this category have already identified critical elements for the acceleration of transition processes. Therefore, what we focused on was to understand how people adapt to these innovations. We believe that these elements can help to explain the roots of SCA in processes of innovation.

We identified four elements of SCA. These elements are interrelated because only by firing together they can explain and lead transition processes. (1) Mirroring and group imitation are linked to the practices that the authors named "replication." It is also linked to "partnering" as while we mirror others, we also interact with them and establish a connection. This leads us to a second element, (2) social group formation related to partnering and networking. In sustainability transition initiatives, new networks and groups form around old ones. Therefore, it is crucial to build relationships with diverse actors and extend the network. From here, we then move to the (3) sense of belonging, which is needed for upscaling and continuous mirroring (Spatiotemporal dimensions). Finally, as the authors have pointed out, creating and capitalizing on a collective narrative (i.e., values, rituals, practices and so on) is crucial for strengthening the transition initiative and keep the diffusion momentum. (4) Adaptation comes last when the novel sociocultural and institutional aspects generated in the phases of niche pruning and acceleration (e.g., new practices, rituals, values, frames and so on) have been embedded in the existing governance structure. However, adaptation starts at an earlier stage, through mirroring and group formation, when the basis 


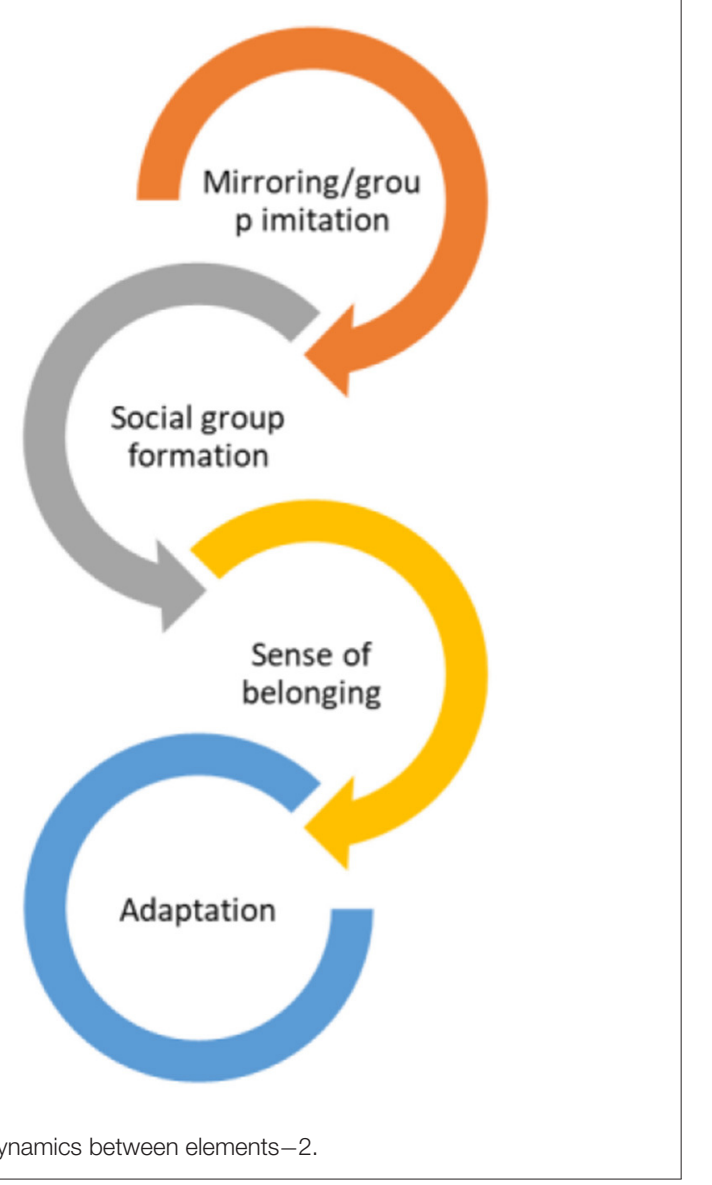

FIGURE 4 | Dynamics between elements-2.

for building a solid layer of trust, acceptance, responsibility, and a sense of local ownership is laid down (Figure 4).

\section{Sociocultural Adaptations in High and Low Tech}

Most of the studies found in the searches, including UA, technology or UA and sustainability transitions, draw the link between different types of UA (e.g., rooftops, vertical farming, community gardens and so on) with high-tech (as remote sensing). They provide data on land cover and building use, communication technology, or high-tech to use in the cultivation-production of food (e.g., aquaponics). These studies stress that adopting new and greener technologies in urban agriculture will enable the transition toward more sustainable and circular cities. In the future, the adoption of these technologies has the potential to ensure food supply (or food security) and its sustainability, equal access to quality food and reduce the stress on agricultural soils and land (Anthopoulos and Vakali, 2012; Cicekli and Barlas, 2014; Mancebo, 2016; Ramaswami et al., 2016; de Amorim et al., 2019; Jürkenbeck et al., 2019; van de Vlasakker and Veen, 2020). These studies emphasize the within scale dynamics and the temporal dynamics concerning the use of high-tech to lead to future sustainable improvements in UA.

\section{Data Gathering}

Authors such as Ramaswami et al. (2016) do not specifically address any type of technology adoption. They instead emphasize the necessity of considering the factors that shape the cities' infrastructure design (i.e., the evolving interaction between physical space and human activity) before devising ways and tools to make cities more circular and sustainable, e.g., via urban agriculture. The rationale is that new technologies, products and ways of producing food will be influenced by the cities' economic, energy, socio-infrastructural, food systems' structure and transportation design. This thus requires that the diverse urban landscape is taken into consideration during the planning phases. Urban planners are using numerous technologies (e.g., remote sensing) to visualize and understand the structure of cities. Urban planners seek not only to focus on the standard measures such as population density and diversity but also "[...] on understanding self-similarity across scales (from blocks to neighborhoods to cities) and patterns of social segregation (e.g., of migrant and informal populations in a city). Urban form represents the foundation upon which infrastructure develops, shaping energy and material use; access to and contiguity of water bodies, green space, and other critical ecosystems; and urban equity and well-being." (Biggs et al., 2010: p. 940).

On a similar perspective, Anthopoulos and Vakali (2012) suggest using remote sensing or airborne sensors for identifying built area characteristics in cities and decide where it is best to install rooftop greenhouses. "The importance of these areas in the concept of the UA is based on the frequent homogeneous [and suitable] characteristics of buildings: the buildings have similar heights and are built at low densities, which prevents buildings from overshadowing nearby rooftops. They are also homogeneous in terms of materials and shapes, having typically much larger floor plans than other type of buildings" (Schwab et al. 2018: p. 495). Rooftop greenhouses can improve cities' energy, production, and environmental performance by valorising unused space (Anthopoulos and Vakali, 2012). The use of technologies such as hydroponic and aeroponic will reduce the structural load of buildings, stress on soils and land in rural areas, and increase the food independence of cities.

\section{Information Sharing}

de Amorim et al. (2019) draw attention to the risks and vulnerabilities of cities to climate change. They are mainly concerned with food insecurities, urban inequalities (e.g., between different ethnic groups) and human health. Climatesmart agriculture is identified as one of the critical solutions to these issues. In climate-smart agriculture, technological innovation is essential but not at the expense of social actions, collaboration, communication, and environmental sustainability. In this perspective, the authors define the industrial revolution 4.0 as aimed "[...] to remove planning, control and decision centralization regarding production and consumption, introducing the Internet of Things (IoT) concept in industrial application scenarios [...] The advances it promoted increase productivity by answering to customers' needs" (Anthopoulos and Vakali 2012: 
p. 87). Actors of the industrial revolution 4.0 use various urban farming technologies (e.g., from spatial farming to LED-based artificial farming, etc.) to secure food production and supply in urban areas. Moreover, communication technologies can also bring customers and producers closer to local food systems, thus promoting market adjustments, lower environmental footprint and increased collaboration (Anthopoulos and Vakali, 2012). This study reflects on the local scale and future time dimensions.

\section{Embedding}

Mancebo (2016) must also be added within this category because he addresses high-tech urban agriculture vs. low-tech urban agriculture. Mancebo (2016) motto is that it takes inhabitants to make a city rather than just architects, engineers and politicians. First, the author twists our attention to forms of local ownership that can boost technology adaptation and embeddedness in every specific urban context. Second, to a comeback to more traditional types of urban agriculture (e.g., community gardens) rather than high-tech sustainable technologies such as aquaponics, vertical farming. Third, again, the emphasis is on within scale dynamics. "When trying to determine if urban agriculture may contribute to a sustainable future, the primary question to ask is: Will this agriculture be at the service of the inhabitantsand not the other way around" (Orsini et al. 2017: p. 12). This question invites us to consider that urban agriculture is not just about food and global warming. Instead, it is also and, most importantly, about social innovations that improve the quality of life, relationships between people and neighbors, social inclusion, building familiar narratives and values around green and sustainable lifestyles. In this perspective, urban agriculture could help bring people together and reconstruct "[...] some kind of urban commons. Such an urban agriculture should be considered as a common good, bringing people together and reshaping the whole urban fabric." (Orsini et al. 2017: p. 13). Similarly, Jürkenbeck et al. (2019) stress the importance of emphasizing the function and advantages of vertical farming, such as sustainability and provision of locally fresh produce. Raising awareness among consumers on these favorable aspects is therefore crucial. "The path analysis of the extended technology acceptance model reveals that the main path starts at the perceived sustainability through perceived usefulness, continues to the attitude toward buying, and finalizes in the behavioral intention to buy" (p. 16).

Alongside van de Vlasakker and Veen (2020) focus on the embedding of high-tech-indoor gardening into a local nursing home for older people and how this technology has led to the transformation of existing cooking and eating practices also the constitutive meaning and competencies of these changes. The study suggests that the awareness of people living and working in nursing homes concerning the benefits of consuming fresh and healthy food has increased since the initiative's beginning. Moreover, cooks have acquired new competencies and knowledge, e.g., about cooking fresh food and retaining nutrients. "As argued, new practices have a higher chance of being adopted when they carry recognizable and familiar elements. Indeed, the new practice of indoor gardening is more easily performed by

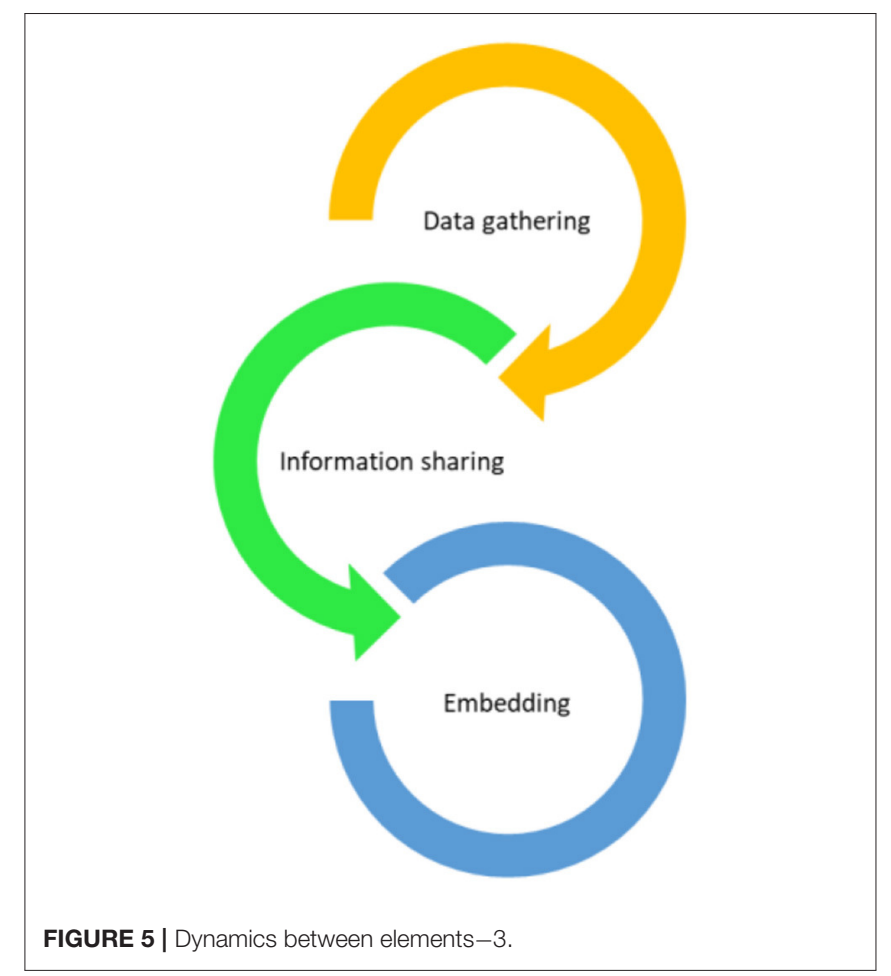

employees who are more confident with related practices, such as gardening/harvesting and cooking" (p. 13).

\section{In Sum}

We identified three fundamental elements ensuing from this literature review. (1) Data gathering is adopted to increase our knowledge of the situation, explore new issues and identify new and more suitable solutions. High-tech is a crucial component of data gathering in urban settings. (2) Information sharing is critical when it comes to building common knowledge and narrative. In more practical terms, it helps to bring together the needs and visions of different actors, thus holding the potential for a dynamic market change. (3) We again deal with embeddedness because local inhabitants must own whatever technology solution we choose. Trust is a critical aspect of the embedding element of SCA. Embedding allows respecting the local inhabitants' existing frames, practices, and rituals by giving them time to transition and adopt different ways of doing things, e.g., cultivating food in urban areas.

\section{Third Summation}

From the studies within this category, we have learned that high-tech can support a better adaptation of urban agriculture modes and technologies by using, for instance, remote sensing. Remote sensing can help us visualize the link between the urban physical space and its use by human activity through the lenses of social differences, e.g., rich and poor, elderlies and young people, and so on. Additionally, ITC technologies can help to reduce the physical, psychological, and social distance between 
different actors. Lastly, when choosing among the diverse urban agriculture options, we should be mindful that green technologies are not necessarily those that will deliver the highest sustainability performance or sustainability outcomes. Moreover, the goal of green urban agriculture should not just be reducing emissions but also creating opportunities for social interaction, social empowerment, and network building. Therefore, we may need to consider that more low-tech options could be a win-win solution to meet different but interrelated social and environmental goals. These studies locate UA within scale dynamics and a future time-span dimension (Figure 5).

\section{CONCLUDING DISCUSSION}

This scoping review has shed light on the blind spot of SCA in the European research on UA and contributed to fill the conceptualmethodological toolbox to understand and explore the elements, complexity, and dynamics of this phenomenon.

Based on the scoping review study results, three points demand particular attention in our concluding discussion. First, the literature on socio-ecological resilience and socio-technical transitions, more than others (i.e., category 1 and 2), deals with SCA, although these studies do not refer to it as such. Second, mainly through the studies in the category 1 and 2 , we have learned about some of the crucial elements of SCA, such as mirroring, sense of belonging, and social group formation. Third, this literature relates more consistently to the within and across-scales dynamics and temporal dimensions. Thus, by scanning this existing literature, we could find the foundations and empirical evidence for strengthening the discussion and role of SCA elements in ensuring just and respectful transition processes and adaptation to climate change. We may hypothesize that socio-technical and socio-ecological innovations are essential to social adaptation to climate change on a more general level.

Additionally, our analysis shows links between elements within and across categories, as illustrated in the Venn Diagram (Figure 2). For instance, sharing (incl. information sharing) and embedding go across themes indicating that these may be fundamental elements for SCA in the European UA sustainability transition. Sharing and embedding help diffuse knowledge, create new relationships, diversify the actors and knowledge network basis, etc. Diversification and social group formation (as a proxy of partnering and networking) precede the previous ones and are a basis for the elements in other categories (e.g., sense of belonging, social memory formation, and adaptation and embedding). This finding sheds some light on the dynamics of sociocultural adaptation to climate change in urban agriculture systems. It seemingly points out that sociocultural adaptation is based on the human beings' necessities to live in groups, share and build shared norms, practices and habits that are passed on generation after generation and are the basis of societal evolution and persistence (as also stressed by the studies in category 1 ).

There is also one underlying common theme, i.e., the necessity of sharing-which builds on mirroring and helps move toward embedding/social group formation. Mirroring is a crucial biological element in human beings and is driven by the necessity of learning and belonging to a community. Communities are not necessarily time and spatially bounded but can be extended beyond the local. Any initiative, policy or intervention aimed at boosting social resilience, e.g., green technology innovation (i.e., adoption and diffusion stages), should build on these core human and social necessities for success. Initiatives that can draw on an existing or to-bebuilt common sense shared values and meaning and then capitalize on building more robust networks. The support to cross-fertilization initiatives will be more successful than policies that only concentrate on economic incentives or environmental taxes/constraints. Decision-makers should capitalize on the local embeddedness of UA initiatives (e.g., reliance on local natural and human resources). This could be done by supporting creating fora and opportunities (e.g., living labs, events) for people to share and co-design innovative solutions suitable to the context and go beyond the local borders (spatial scale). Moreover, political support is necessary to network, upscale and diffuse sustainable and just practices around new UA technologies.

Additionally, although this paper is not the proper ground for theoretical discussions, we believe that we could make a theoretical hypothesis to be further explored in new studies. Notably, the elements of sociocultural adaptation in EU UA could be thought of as the socio-biological mechanisms through which SCA occurs. They exist because social and biological structures are inevitably and mutually interlinked. Therefore, these mechanisms of SCA could be used comparably to analyze SCA in other cases to see if they recur and persist in other sociocultural contexts. Dealing with SCA bears whether we need to move beyond the social sciences to understand its elements, e.g., why is SCA possible? What enables SCA elements? We answer that there is a need to bridge the social sciences with other disciplines, such as human behavioral biology and neurobiology, to understand SCA elements in urban agriculture and food system sustainability transitions. In reviewing Bristol's book (Bristol, 1915), Woolston stated that by "social adaptation is meant "[...] such a state of adjustment between an organism or a social group and its environment as is favorable to existence and growth, or the process by which such unity comes into, and continues in, this favorable relation. The environment is both physical, or material, and social, or spiritual" (p. 311). We can generally classify the various structures (or conditions) that underlie adaptation processes in the bioeconomy as biological, sociocultural and technological (Grin et al., 2011; Mingers, 2014; Chaffin and Gunderson, 2016; Sapolsky, 2017). These structures represent the contextual conditions that enable, influence, and shape SCA elements in sustainability transitions. The "observed phenomenon," in this case, is the new and adapted sociality related to UA systems-growing and living in cities.

Our point of departure was based on a sensitizing definition of SCA, notably changes or adjustments in critical dimensions, such as culture, practices, norms, perceptions, and frames. After our review, this definition may need to be expanded to cover 
the complexity of SCA. Inspired by Hegnes (2013), our new suggestion is that an understanding of SCA dynamics occurs in continuous meaning-making processes of ideas and mindsets. These processes are related to and influenced by structures of social relations, psychological conditions, physical objects, and how cultural complexity is woven into society in general and characterized by a relative autonomy in shaping actions and institutions.

Despite its elaborate approach, the scoping review has limitations imposed by the spatial-linguistic boundary. Notably, taking the European Union combined with English-written literature leads to uniformizing the cultural, social, and institutional contexts. However, we believe that for our stated goal of exploration, i.e., shed light and systematize some of the complexity and dynamics of SCAs in European urban agriculture. This "uniformization" does not jeopardize the analytical potential of the results for category construction. Furthermore, we do not aim to generalize our results to all contexts. Instead, we aim to provide an initial and explorative categorization of SCAs and elements that can be deployed, tested, and validated in future research on sustainability transitions.

In conclusion, our study provided first, hands-on evidence of how elements of SCA play out in the long-term transition and climate adaptation processes, as well as a basis to design policies and intervention strategies that can capitalize on these elements. Thus, the study is a first step toward filling the research gap concerning social adaption in UA and food systems transition. However, additional work is needed to, on the one hand, develop a middle-range theory of SCA mechanisms by linking social science with other disciplines, such as neurobiology and human behavioral biology. On the other hand, it is biological structures (neuronal firing in areas of the brain) that enable specific human abilities, such as the mirroring element, which are at the basis of

\section{REFERENCES}

Adger, W. N. (2000). Social and ecological resilience: are they related? Prog. Hum. Geogr. 24, 347-364. doi: 10.1191/0309132007015 40465

Alvesson, M., and Sköldberg, K. (2009). Reflexive Methodology: New Vistas for Qualitative Research (Second). London: SAGE Publications. Available online at: https://books.google.be/books/about/Reflexive_Methodology.html? $\mathrm{id}=J r x x H r 3 L k s Q C a n d r e d i r \_e s c=y$ (accessed October 3, 2021).

Anthopoulos, L. G., and Vakali, A. (2012). Urban planning and smart cities: interrelations and reciprocities. Lect. Notes Comput. Sci. 7281 LNCS, 178-189. doi: 10.1007/978-3-642-30241-1_16

Arksey, H., and O'Malley, L. (2007). Scoping studies: towards a methodological framework. Int. J. Soc. Res. Methodol. 8, 19-32. doi: 10.1080/1364557032000119616

Barnes, J., Durrant, R., Kern, F., and MacKerron, G. (2018). The institutionalisation of sustainable practices in cities: how initiatives shape local selection environments. Environ. Innov. Soc. Trans. 29, 68-80. doi: 10.1016/j.eist.2018.04.003

Barthel, S., Folke, C., and Colding, J., (2010). Social-ecological memory in gardening: retaining the capacity for management of ecosystem services. Global Environ. Change 20:255-265.

Barthel, S., and Isendahl, C. (2013). Urban gardens, agriculture, and water management: sources of resilience for long-term food security in cities. Ecol. Econ. 86, 224-234. doi: 10.1016/j.ecolecon.2012. 06.018 social group formation, sense of belonging, SCA etc. (Iacoboni, 2008, 2009; Siegel, 2015; Sapolsky, 2017). "By helping us recognize the actions of other people, mirror neurons also help us to recognize and understand the deepest motives behind those actions, the intentions of other individuals" (Iacoboni, 2008: p. 6). Therefore, we could assume that these elements of social adaption are mechanisms that ensure and link biological and social structures (e.g., mirror neurons make mirroring/imitation possible, thus enabling other social behaviors and structure formation, such as social group formation, the institutionalization of standard practices into rules and norms etc.). Thus, regularities or recurrent mechanisms in SCA throughout different contexts could be found at the level of these elements or mechanisms. On the other hand, further research is needed to test and validate this assumption about mechanisms of SCA, for instance, through case-study research.

\section{AUTHOR CONTRIBUTIONS}

All authors listed have made a substantial, direct and intellectual contribution to the work, and approved it for publication.

\section{FUNDING}

This research was funded by the Horizon 2020 (European Union Framework Programme for Research and Innovation) project SiEUGreen, Grant no. 774233.

\section{ACKNOWLEDGMENTS}

The authors are grateful for constructive and insightful comments by the two reviewers, which helped to structure and nuance the paper.

Berthet, E. T., and Hickey, G. M. (2018). Organizing collective innovation in support of sustainable agro-ecosystems: the role of network management. Agric. Syst. 165, 44-54. doi: 10.1016/j.agsy.2018.05.016

Biggs, R., Westley, F. R., and Carpenter, S. R. (2010). Navigating the back loop: fostering social innovation and transformation in ecosystem management. Ecol. Soc. 15:28. doi: 10.5751/ES-03411-150209

Boossabong, P. (2019). Governing Bangkok's city food system: engaging multistakeholders for smart, sustainable and inclusive growth. City Cult. Soc. 16, 52-59. doi: 10.1016/j.ccs.2018.05.001

Bristol, L. M. (1915). Social Adaptation; a Study in the Development of the Doctrine of Adaptation as a Theory of Social Progress, (Book, 1915) [WorldCat.org] (Paper). Cambridge, MA: Harvard University Press. Available online at: https:// www.worldcat.org/title/social-adaptation-a-study-in-the-development-ofthe-doctrine-of-adaptation-as-a-theory-of-social-progress/oclc/1457368 (accessed October 3, 2021).

Camps-Calvet, M., Langemeyer, J., Calvet-Mir, L., and Gómez-Baggethun, E. (2015). Sowing resilience and contestation in times of crises: the case of urban gardening movements in Barcelona. Partecipazione e Conflitto. Open J. Soc. Stud. 8, 417-442. doi: 10.1285/i20356609v8i2p417

Celata, F., and Coletti, R. (2018). The policing of community gardening in Rome. Environ. Innov. Soc. Trans. 29, 17-24. doi: 10.1016/j.eist.2017. 09.002

Chaffin, B. C., and Gunderson, L. H. (2016). Emergence, institutionalization and renewal: rhythms of adaptive governance in complex social-ecological systems. J. Environ. Manage. 165, 81-87. doi: 10.1016/j.jenvman.2015. 09.003 
Cicekli, M., and Barlas, N. T. (2014). Transformation of today greenhouses into high technology vertical farming systems for metropolitan regions. J. Environ. Protect. Ecol. 15, 1779-1785. Available online at: https://docs.google.com/a/jep e-journal.info/viewer?a=v\&pid=sites\&srcid=amVwZS1qb3VybmFsLmluZm9 8amVwZS1qb3VybmFsfGd4OjQ1MzQ3ZTRhYzI2YTRINjk

Classens, M. (2015). The nature of urban gardens: toward a political ecology of urban agriculture. Agric. Hum. Values 32, 229-239. doi: 10.1007/s10460-014-9540-4

Corcoran, M. P., and Kettle, P. C. (2015). Urban agriculture, civil interfaces and moving beyond difference: the experiences of plot holders in Dublin and Belfast. Local Environ. 20, 1215-1230. doi: 10.1080/13549839.2015.1038228

Cote, M., and Nightingale, A. J. (2012). Resilience thinking meets social theory. Prog. Hum. Geogr. 36, 475-489. doi: 10.1177/0309132511425708

Curry, N., Reed, M., Keech, D., Maye, D., and Kirwan, J. (2014). Urban agriculture and the policies of the European Union: the need for renewal. Span. J. Rural Dev. 5, 91-106. doi: 10.5261/2014.ESP1.08

Cvejić, R., Železnikar, Š., Nastran, M., Rehberger, V., and Pintar, M. (2015). Urban agriculture as a tool for facilitated urban greening of sites in transition: a case study. Urbani Izziv 26, S84-S97. doi: 10.5379/urbani-izziv-en-2015-26-supplement-006

de Amorim, W. S., Borchardt Deggau, A., do Livramento Gonçalves, G., da Silva Neiva, S., Prasath, A. R., and Salgueirinho Osório de Andrade Guerra, J. B. (2019). Urban challenges and opportunities to promote sustainable food security through smart cities and the 4 th industrial revolution. Land Use Policy 87:104065. doi: 10.1016/j.landusepol.2019.104065

Durrant, R., Barnes, J., Kern, F., and Mackerron, G. (2018). The acceleration of transitions to urban sustainability: a case study of Brighton and Hove. Eur. Plan. Stud. 26, 1537-1558. doi: 10.1080/09654313.2018.1489783

European Commission (2020). Farm to Fork Strategy. For a Fair, Healthy, and Environmentally-Friendly Food System. Brussels: European Commission.

Fastenrath, S., and Braun, B. (2018). Lost in transition? directions for an economic geography of urban sustainability transitions. Sustainability (Switzerland) 10, 11-13. doi: 10.3390/su10072434

Folke, C., Colding, J., Berkes, F., (eds.). (2003). "Synthesis: building resilience and adaptive capacity in social-ecological systems," in Navigating SocialEcological Systems: Building Resilience for Complexity and Change, (Cambridge: Cambridge University Press), 352-387.

Giacchè, G., and Porto, L. (2018). The incredible Edible Movement: people power, adaptation, and challenges in Rennes (France) and Montreal (Canada). Nat. Cult. 13, 92-112. doi: 10.3167/nc.2018.130105

Gorissen, L., Spira, F., Meynaerts, E., Valkering, P., and Frantzeskaki, N. (2018). Moving towards systemic change? investigating acceleration dynamics of urban sustainability transitions in the Belgian City of Genk. J. Clean. Prod. 173, 171-185. doi: 10.1016/j.jclepro.2016.12.052

Grin, J., Rotmans, J., and Schot, J. (2011). On patterns and agency in transition dynamics: Some key insights from the KSI programme. Environ. Innov. Soc. Trans. 1, 76-81. doi: 10.1016/j.eist.2011.04.008

Gulyas, B. Z., and Edmondson, J. L. (2021). Increasing city resilience through urban agriculture: challenges and solutions in the global north. Sustainability 13:1465. doi: $10.3390 /$ su13031465

Hearn, G., Collie, N., Lyle, P., Choi, J. H. J., and Foth, M. (2014). Using communicative ecology theory to scope the emerging role of social media in the evolution of urban food systems. Futures 62, 202-212. doi: 10.1016/j.futures.2014.04.010

Hegnes, A. W. (2013). Kulturelt tilpasningsarbeid. Innføring, forvaltning og bruk av merkeordningen Beskyttede betegnelser i Norge (Cultural adaptation workimplementation, administration and the use of geographical indications in norway) (doctoral thesis). Department of Sociology and Human Geography, Faculty of Social Sciences, University of Oslo, Oslo, Norway. Available online at: http://urn.nb.no/URN:NBN:no-38793

Hennchen, B., and Pregernig, M. (2020). Organizing joint practices in urban food initiatives - a comparative analysis of gardening, cooking, and eating together. Sustainability 12:4457. doi: 10.3390/su12114457

Huston, S., Rahimzad, R., and Parsa, A. (2015). "Smart” sustainable urban regeneration: Institutions, quality and financial innovation. Cities 48, 66-75. doi: 10.1016/j.cities.2015.05.005

Iacoboni, M. (2008). Mirroring People: The Science of Empathy and How We Connect With Others by Marco Iacoboni (Paperback). London: Picador.
Available online at: https://www.goodreads.com/book/show/6680624mirroring-people (accessed October 3, 2021).

Iacoboni, M. (2009). Imitation, empathy, and mirror neurons. Annu. Rev. Psychol. 60, 653-670. doi: 10.1146/annurev.psych.60.110707.1 63604

Jürkenbeck, K., Heumann, A., and Spiller, A. (2019). Sustainability matters: consumer acceptance of different vertical farming systems. Sustainability 11:4052. doi: 10.3390/su11154052

Mancebo, F. (2016). Urban agriculture, commons and urban policies: scaling up local innovation. Chall. Sustain. 4, 10-19. doi: 10.12924/cis2016.040 10010

McEldowney, J. (2017). Urban Agriculture in Europe: Patterns, Challenges, and Policies. Brussels: European Parliament Think Tank. Available online at: https://www.europarl.europa.eu/thinktank/en/document.html?reference= EPRS_IDA(2017)614641 (accessed October 3, 2021).

Mingers, J. (2014). Systems Thinking, Critical Realism and Philosophy: A Confluence of Ideas (Ontological Explorations) First (Kin). New York, NY: Taylor and Francis.

Munn, Z., Peters, M. D. J., Stern, C., Tufanaru, C., Mcarthur, A., and Aromataris, E. (2018). Systematic review or scoping review? guidance for authors when choosing between a systematic or scoping review approach. BMC Med. Res. Methodol. 18, 1-7. doi: 10.1186/s12874-0180611-x

Oda, K., Rupprecht, C. D. D., Tsuchiya, K., and McGreevy, S. R. (2018). Urban agriculture as a sustainability transition strategy for shrinking cities? Land use change trajectory as an obstacle in Kyoto city, Japan. Sustainability (Switzerland) 10:1048. doi: 10.3390/su100 41048

Ogburn, W. F. (1922). Social Change With Respect to Culture and Original Nature. New York, NY: Viking Press.

Orsini, F., Dubbeling, M., De Zeeuw, H., and Gianquinto, G. (2017). Urban Agriculture: Rooftop Urban Agriculture. (F. O. D. de Z. Gianquinto, Ed.) (Paper). Cham: Springer.

Partalidou, M., and Anthopoulou, T. (2017). Urban allotment gardens during precarious times: from motives to lived experiences. Sociol. Ruralis 57, 211-228. doi: 10.1111/soru.12117

Pham, M. T., Raji,ć, A., Greig, J. D., Sargeant, J. M., Papadopoulos, A., and McEwen, S. A. (2014). A scoping review of scoping reviews: advancing the approach and enhancing the consistency. Res. Synth. Methods 5:371. doi: 10.1002/jrsm.1123

Ramaswami, A., Russell, A. G., Culligan, P. J., Rahul Sharma, K., and Kumar, E. (2016). Meta-principles for developing smart, sustainable, and healthy cities. Science 352, 940-943. doi: 10.1126/science.aa f7160

Russo, A., Escobedo, F. J., Cirella, G. T., and Zerbe, S. (2017). Edible green infrastructure: an approach and review of provisioning ecosystem services and disservices in urban environments. Agric. Ecosyst. Environ. 242, 53-66. doi: 10.1016/j.agee.2017.03.026

Sanyé-Mengual, E., Anguelovski, I., Oliver-Sol,à, J., Montero, J. I., and Rieradevall, J. (2016). Resolving differing stakeholder perceptions of urban rooftop farming in Mediterranean cities: promoting food production as a driver for innovative forms of urban agriculture. Agric. Hum. Values 33, 101-120. doi: 10.1007/s10460-015-9594-y

Sanyé-Mengual, E., Specht, K., Grapsa, E., Orsini, F., and Gianquinto, G. (2019). How can innovation in urban agriculture contribute to sustainability? a characterization and evaluation study from five western european cities. Sustainability 11:4221. doi: 10.3390/su111 54221

Sapolsky, R. M. (2017). Behave: The Biology of Humans at Our Best and Worst. New York, NY: Penguin Press.

Schoen, V., Caputo, S., and Blythe, C. (2020). Valuing physical and social output: a rapid assessment of a london community garden. Sustainability 12:5452. doi: 10.3390/su121 35452

Schwab, E., Caputo, S., and Garcia, J. H. (2018). Urban agriculture: models in circulation from a critical transnational perspective authors: eva schwab, silvio caputo and jaime hernandez garcia. Landsc. Urban Plan. 170, 15-23. doi: 10.1016/j.landurbplan.2017.09.012 
Siegel, D. J. (2015). The Developing Mind: How Relationships and the Brain Interact to Shape Who We Are. (I. Edition, Ed.). New York, NY: Guilford Press.

Sorokin, P. (2006 [1957]). Social and Cultural Dynamics: A Study of Change in Major Systems of Art, Truth, Ethics, Law, and Social Relationships (Revised and Abridged in One Volume by the Author.). New Brunswick, NJ: Transaction Publishers.

Taylor, J. R., and Lovell, S. T. (2014). Urban home food gardens in the Global North: research traditions and future directions. Agric. Human Values 31, 285-305. doi: 10.1007/s10460-013-9475-1

Thornbush, M. (2015). Urban agriculture in the transition to low carbon cities through urban greening. AIMS Environ. Sci. 2, 852-867. doi: 10.3934/environsci.2015.3.852

Topal, H. F., Hunt, D. V. L., and Rogers, C. D. F. (2021). Exploring urban sustainability understanding and behaviour: a systematic review towards a conceptual framework. Sustainability 13:1139. doi: 10.3390/su130 31139

van de Vlasakker, P. C. H., and Veen, E. J. (2020). Effects of high-tech urban agriculture on cooking and eating in dutch nursing homes. Sustainability 12:5379. doi: 10.3390/su12135379

Woolston, H. B. (1917). Social adaptation: a study in the development of the doctrine of adaptation as a theory of social progress. Am. J. Theol. 21, 311-313. doi: $10.1086 / 479837$
Zimmerer, K. S., Bell, M. G., Chirisa, I., Duvall, C. S., Egerer, M., Hung, P.Y., et al. (2021). Grand challenges in urban agriculture: ecological and social approaches to transformative sustainability. Front. Sustain. Food Syst. 5:668561. doi: $10.3389 /$ fsufs. 2021.668561

Conflict of Interest: The authors declare that the research was conducted in the absence of any commercial or financial relationships that could be construed as a potential conflict of interest.

Publisher's Note: All claims expressed in this article are solely those of the authors and do not necessarily represent those of their affiliated organizations, or those of the publisher, the editors and the reviewers. Any product that may be evaluated in this article, or claim that may be made by its manufacturer, is not guaranteed or endorsed by the publisher.

Copyright (c) 2021 Cavicchi and Hegnes. This is an open-access article distributed under the terms of the Creative Commons Attribution License (CC BY). The use, distribution or reproduction in other forums is permitted, provided the original author(s) and the copyright owner(s) are credited and that the original publication in this journal is cited, in accordance with accepted academic practice. No use, distribution or reproduction is permitted which does not comply with these terms. 